\title{
DEFINING COMPETITIVITY OF AN ABSORBING MARKOV CHAIN
}

\author{
V. I. Teslenko ${ }^{1}$, O. L. Kapitanchuk ${ }^{2}$ \\ Bogolyubov Institute for Theoretical Physics, National Academy of Sciences of Ukraine, \\ Metrologichna St., 14-B, Kyiv, 03680, Ukraine \\ e-mails: ${ }^{1}$ vtes@bitp.kiev.ua, ${ }^{2}$ alkapt@bitp.kiev.ua
}

(Received 30 January, 2015)

\begin{abstract}
The problem of defining the competitivity for the different states of an absorbing Markov chain is formulated in a continuous time framework. Chain states are associated with the modules of locally equilibrated, fluctuating energy levels formed due to strong adiabatic interactions within a nonequilibrium system being nonadiabatically coupled weakly to the equilibrium environment. Using a microscopic approach to the description of time evolution of the density matrix of the whole system, we first reduce the corresponding Liouville-von Neumann equation to a master equation for diagonal elements of the density matrix of a nonequilibrium system, then provide a calculus of involved chaotic and stochastic averages with the supposed initial and completed energy-level distributions, and finally arrive at the kinetic equation for the population of aggregated chain states. The equation is in detail balanced by respective transition probabilities being well defined for all the differences between energies and dimensionalities of the chain state modules. For the case of an absorbing Markov chain, this makes it possible to define the competitivity for different modular states, as an inverse slope of log odds of normalized peaks of their population with respect to log of various input transition probabilities set to be free in the wide limits.
\end{abstract}

Key words: nonequilibrium systems, nonstationary kinetics, irreversible processes, absorbing Markov chain, competitiveness.

PACS number(s): 05.70.Ln, 02.50.Ga, 05.40.-a

\section{INTRODUCTION}

There exists no single definition of competitivity, which can be applicable for all realizations of transition processes in the natural, life, economic and information chains. The finite state supply chain lies at the heart of interpreting a time evolution of many kinetic processes. For instance, a Markov chain is widely used for modeling the multistage dynamics of multidimensional states in physics [1], chemistry [2] and biology [3]. The same is true for the decision making and strategy development chain models to implement in informatics [4] and economy [5]. However, despite a great interest in lots of applications, the definitions of competitiveness known to date for Markov chain states remain unstrict and do not lend themselves to any systematic observations.

In general, competitiveness of a particular system depends on the number of its characteristics, which as a rule are interrelated and cannot be considered in isolation. The main factors include: the initial conditions for population of input states, the time evolution of population of functionally significant intermediate and output states, and the kinetic coefficients providing a growth of population with the probabilities to transit some states of interest toward others due to their weak competitive coupling with the environment. Both the former and the latter factors are usually maintained externally or imposed conventionally, rather the central factor is found as a solution to the problem, given the two. However, because of lack of the detailed information about the microscopic states of Markov chains, using the heuristic state-based models for evaluation of the insufficient- ly understood quantities such as competitivity is largely inconsistent. In this situation, which is often the case for multiatomic and macromolecular systems, making a consideration that will be compatible with microscopic and phenomenological descriptions must be based upon the general physical principles. Therefore, it is necessary first to correctly define, for the whole system, a set of orthonormal states and introduce an equation for their operator transformations and further average that equation over irrelevant states to obtain the reduced balance kinetic equation for the population of relevant states, and only then calculate the between-state transition rates with allowing their values to vary freely in the extended range.

Following the arguments above, contained in part in the previous papers [6-9], this work aims at the attempt to provide a microscopically rigorous formulation of the problem of competitivity for the general case of a continuous time Markov chain having the absorbing states. Here, the basic difficulty is entailed by the fact that an absorbing Markov chain represents the non-ergodic process of memoryless transitions between the non-recurrent transient states that satisfy only zero stationary conditions. Therefore, in this case, the measures of interest are restricted to mainly cumulative probabilities characterizing the time a chain spends in non-absorbing states before an absorbing state is ultimately reached [4]. However, in the nonstationary irreversible systems, there are also other characteristic features of the transitory dynamics during which the states' population increase, peak and decline. Just with respect to the peaks of the population the competitivity of one state in regard to another can be defined most accurately. To substantiate 


\section{I. TESLENKO, O. L. KAPITANCHUK}

this statement, Section II of the paper sets forth a microscopic description of the nonequilibrium system with fluctuating energy levels weakly coupled to the equilibrium environment. Sections III and IV are devoted to a derivation of the corresponding kinetic equation for diagonal elements of the density matrix of the nonequilibrium system and the master equation for the population of the different modules of a system's states, respectively. Rate constants of transitions between the modules corresponding to the aggregated transition probabilities in an absorbing Markov chain are found in Section V. Towards the end, Section VI defines the competitivity of Markov chain states and presents some discussion.

\section{MICROSCOPIC BACKGROUND}

Consider the microscopic formulation allowing for a finite state Markov chain to be mapped from an infinite state condensed phase system onto its discrete (quantum) and quasi-continuous (nuclear) coordinates. For definiteness, let us regard a condensed phase system as the whole system characterized by a finite set of quantum states (energy levels) and an infinite set of vibration excitations (phonons). Let the former set comprise a nonequilibrium system $(\mathrm{S})$ which is nonadiabatically coupled weakly to an equilibrium environment constituting the latter set, thus playing a role of the heat bath (B) for the S. Let the energy levels $E_{j}(t)=E_{j}+\hbar \Omega_{j}(t)$ of the $\mathrm{S}$ adiabatically fluctuate round their means $E_{j}$ with the stochastic frequencies $\Omega_{j}(t)$ while the quantum states $|j=0,1, \ldots, J\rangle$ are stationary ( $\hbar$ is the Planck constant). Then the Hamiltonian of the whole closed system "the $\mathrm{S}$ + the B + their weak coupling" reads

$$
\begin{aligned}
H(t) & =\sum_{j}\left\{E_{j}(t)+\sum_{\lambda}\left[\kappa_{j}^{(\lambda)}\left(\beta_{\lambda}^{+}+\beta_{\lambda}\right)\right]+H_{\mathrm{B}}\right\}|j\rangle\langle j| \\
& +\sum_{j j^{\prime}} V_{j j^{\prime}}\left(1-\delta_{j j^{\prime}}\right)|j\rangle\left\langle j^{\prime}\right|
\end{aligned}
$$

where $\kappa_{j}^{(\lambda)}$ are the parameters of nuclei displacements $\left(\beta_{\lambda}^{+}-\beta_{\lambda}\right)$ along $\lambda$ th normal coordinates of the $\mathrm{B}$ with the Hamiltonian

$$
H_{\mathrm{B}}=\sum_{\lambda} \hbar \omega_{\lambda}\left(\beta_{\lambda}^{+} \beta_{\lambda}+1 / 2\right)
$$

provided that $\omega_{\lambda}$ represent the corresponding vibration modes (phonon frequencies) with $\beta_{\lambda}^{+}$or $\beta_{\lambda}$ being the operators of creation or annihilation of the respective phonons.

Thus, the whole system is in fact decomposed into two parts. The first part is the $\mathrm{S}$ with adiabatic (stationary) states $|j\rangle$ and stochastic energies $E_{j}(t)$. The second part is the $\mathrm{B}$ with normal modes $\omega_{\lambda}$. The transitions between the states are determined by the matrix elements $V_{j j^{\prime}}$ in (1) that are modeled depending on the intensity of the coupling of the $\mathrm{S}$ to the $\mathrm{B}$. In a weak coupling limit, it is possible to expand the coupling over $\left(\beta_{\lambda}^{+}-\beta_{\lambda}\right)$ so that linear (one-phonon) or nonlinear (multiphonon) terms of expansion are considered as perturbations. In a non-perturbation approach with Holstein's transformation [10], one commonly uses a unitary matrix $U=\exp \left(\sum_{j} u_{j}|j\rangle\langle j|\right)$, where $u_{j}=\sum_{\lambda} g_{j}^{(\lambda)}\left(\beta_{\lambda}^{+}-\beta_{\lambda}\right)$ is the displacement operator of the $j$ th state and $g_{j}^{(\lambda)}=$ $\kappa_{j}^{(\lambda)} / \hbar \omega_{\lambda}$ is a dimensionless coupling. Multiplying (1) from the left by $U$ and from the right by $U^{+}$gives exactly

$$
H(t)=H_{\mathrm{S}}(t)+V+H_{\mathrm{B}}
$$

In this equation, the Hamiltonian of the $\mathrm{S}$

$$
H_{\mathrm{S}}(t)=\sum_{j}\left[\tilde{E}_{j}+\hbar \Omega_{j}(t)\right]|j\rangle\langle j|
$$

specifies the energies $\tilde{E}_{j}=E_{j}-\sum_{\lambda}\left|\kappa_{j}^{(\lambda)}\right|^{2} / \hbar \omega_{\lambda}$ that are adiabatically "dressed" by phonons (the tilda overbar is dropped in the sequel), and

$$
V=\sum_{j j^{\prime}}\left(1-\delta_{j j^{\prime}}\right) V_{j j^{\prime}} \exp \left(u_{j j^{\prime}}\right)|j\rangle\left\langle j^{\prime}\right|
$$

is the operator of nonadiabatic relaxation interaction for transitions between "phonon-dressed" adiabatic states, with $u_{j j^{\prime}}=\sum_{\lambda} g_{j j^{\prime}}^{(\lambda)}\left(\beta_{\lambda}^{+}-\beta_{\lambda}\right)$ being the operator of relative displacements due to the couplings $g_{j j^{\prime}}^{(\lambda)}=\left[\kappa_{j}^{(\lambda)}-\right.$ $\left.\kappa_{j^{\prime}}^{(\lambda)}\right] / \hbar \omega_{\lambda}$.

The main characteristic feature of the microscopic Hamiltonians (1)-(5) is providing an adiabatic refinement of energies $E_{j}(t)$ (4) of the $\mathrm{S}$ with the stochastic additions $\hbar \Omega_{j}(t)$ corresponding to an account of random back actions of the $\mathrm{B}$ on the $\mathrm{S}$ then to follow nonadiabatic transitions between the states (5). Just due to the presence of random fluctuations of adiabatic (stationary) levels $E_{j}$ the process of their nonadiabatic (transient) relaxation becomes irreversible by manifesting itself as a memoryless exchange of phonon excitation energy between the $\mathrm{S}$ and the $\mathrm{B}$. Moreover, making the energy levels fluctuate allows for a straightforward possibility to directly relate an adsorbing Markov chain to a non-recurrent evolution process by introducing the corresponding modular chain states with potentially high or even infinite dimensionality.

\section{KINETIC EQUATION FOR ENSEMBLE-AVERAGED POPULATIONS OF STATES}

Given Hamiltonians (1)-(5), the next step is to describe the dynamics of the density matrix $\rho(t)$ of the whole system following the Liouville-von Neumann quantum evolution equation

$$
\dot{\rho}(t)=-i L(t) \rho(t)
$$

where $L(t)=(1 / \hbar)[H(t), \ldots]$ is the Liouville superoperator related to the stochastic Hamiltonian (1). Since the whole system is decomposed into two parts, the 
$\rho(t)$ factorizes by the equilibrium density matrix $\rho_{\mathrm{B}}=$ $\exp \left(-H_{\mathrm{B}} / k_{\mathrm{B}} T\right) / \operatorname{tr}_{\mathrm{B}} \exp \left(-H_{\mathrm{B}} / k_{\mathrm{B}} T\right)$ of the $\mathrm{B}$ and the nonequilibrium density matrix $\rho_{\mathrm{S}}(t)=\operatorname{tr}_{\mathrm{B}} \rho(t)$ of the $\mathrm{S}$ so that

$$
\rho(t)=\rho_{\mathrm{S}}(t) \rho_{\mathrm{B}}
$$

Therefore, it is sufficient to describe the dynamics in (6) for only the diagonal part of $\rho_{\mathrm{S}}(t)$. To do that, one acts on (6) by the projection operators $\hat{T}_{d}$ and $\hat{T}_{\text {nd }}=I-\hat{T}_{d}$. This gives the coupled set of differential equations for the diagonal $\rho^{(\mathrm{d})}(t)=\hat{T}_{\mathrm{d}} \rho(t)$ and off-diagonal $\rho^{(\mathrm{nd})}(t)=\hat{T}_{\text {nd }} \rho(t)$ parts of the density matrix $\rho(t)$ of the whole system

$$
\left\{\begin{array}{l}
\dot{\rho}^{(\mathrm{d})}(t)=-i \hat{T}_{d} L_{V} \rho^{(\mathrm{nd})}(t), \\
\dot{\rho}^{(\mathrm{nd})}(t)=-i \hat{T}_{\mathrm{nd}}\left[L_{\mathrm{S}}(t)+L_{V}\right] \rho^{(\mathrm{nd})}(t)-i L_{V} \rho^{(\mathrm{d})}(t)
\end{array} .\right.
$$

Substituting the second equation of this system into the first one and owing to the definition of the nonequilibrium density matrix with factorization condition (7) lead to the integral differential equation for $\rho_{\mathrm{S}}^{(\mathrm{d})}(t)$

$$
\dot{\rho}_{\mathrm{S}}^{(\mathrm{d})}(t)=-\int_{0}^{t} d t^{\prime} \hat{\mathrm{M}}\left(t, t^{\prime}\right) \rho_{\mathrm{S}}^{(\mathrm{d})}\left(t^{\prime}\right)
$$

where

$$
\hat{\mathrm{M}}\left(t, t^{\prime}\right)=\operatorname{tr}_{\mathrm{B}}\left\{\hat{T}_{d} L_{V} \hat{D} \exp \left[-i \int_{t^{\prime}}^{t} d \tau \hat{T}_{\mathrm{nd}}\left(L_{\mathrm{S}}(\tau)+L_{V}\right)\right] L_{V} \rho_{\mathrm{B}}\right\}
$$

is the stochastic transition kernel superoperator, and $L_{\mathrm{S}}(t)=(1 / \hbar)\left[H_{\mathrm{S}}(t), \ldots\right], L_{\mathrm{B}}=(1 / \hbar)\left[H_{\mathrm{B}}, \ldots\right]$ and $L_{V}=$ $(1 / \hbar)[V, \ldots]$ are the corresponding Liouville superoperators related to the Hamiltonians of system (4), environment (2) and interaction (5), respectively, with $\hat{D}$ being the Dyson time-ordering operator.

The form of (8), (9) is very convenient for the expansion over a weak interaction $V$. Thus, in the Born approximation constraining the right hand side of $(8)$ to the second-order terms, one has to be restricted in the integrand of the exponent of (9) to only the case of $V=0$. Using this yields

$$
\dot{\rho}_{\mathrm{S}}^{(\mathrm{d})}(t)=-\left(1 / \hbar^{2}\right) \int_{0}^{t} d t^{\prime} \operatorname{tr}_{\mathrm{B}}\left\{\hat{T}_{d}\left[V, U\left(t, t^{\prime}\right)\left[V, \rho_{\mathrm{S}}^{(\mathrm{d})}\left(t^{\prime}\right) \rho_{\mathrm{B}}\right] U^{+}\left(t, t^{\prime}\right)\right]\right\}
$$

where $U\left(t, t^{\prime}\right)=\hat{D} \exp \left[-(i / \hbar) \int_{t^{\prime}}^{t} d \tau\left(H_{\mathrm{S}}(\tau)+H_{\mathrm{B}}\right)\right]$ is the two-time evolution superoperator. By substituting now the $V(4)$ in (10) with the operators $V_{j j^{\prime}} \exp \left(u_{j j^{\prime}}\right)$ considered as perturbations one can easily derive a stochastic equation for the population $p_{j}(t)=$ $\left\langle j\left|\rho_{\mathrm{S}}^{(\mathrm{d})}(t)\right| j\right\rangle$ of the states

$$
\dot{p}_{j}(t)=-\sum_{j^{\prime}} \int_{0}^{t} d t^{\prime}\left[G_{j j^{\prime}}\left(t, t^{\prime}\right) p_{j}\left(t^{\prime}\right)-G_{j^{\prime} j}\left(t, t^{\prime}\right) p_{j^{\prime}}\left(t^{\prime}\right)\right]
$$

Here the two-time kernel

$$
\begin{aligned}
G_{j j^{\prime}}\left(t, t^{\prime}\right) & =\left(2 / \hbar^{2}\right)\left|V_{j^{\prime} j}\right|^{2} \operatorname{Re}\left\{Q_{j j^{\prime}}\left(t-t^{\prime}\right) f_{j j^{\prime}}\left(t, t^{\prime}\right)\right. \\
& \left.\times \exp \left[i\left(\Delta E_{j j^{\prime}} / \hbar\right)\left(t-t^{\prime}\right)\right]\right\}
\end{aligned}
$$

exhibits a stochastic behavior via the random difference frequency $\Omega_{j j^{\prime}}(\tau)=\Omega_{j}(\tau)-\Omega_{j^{\prime}}(\tau)$ involved in the functional

$$
f_{j j^{\prime}}\left(t, t^{\prime}\right)=\exp \left\{i \int_{t^{\prime}}^{t} d \tau \Omega_{j j^{\prime}}(\tau)\right\}
$$

while $\Delta E_{j j^{\prime}} / \hbar=\left(E_{j}-E_{j^{\prime}}\right) / \hbar$ and $Q_{j j^{\prime}}\left(t-t^{\prime}\right)=$ $\operatorname{tr}_{\mathrm{B}}\left\{\rho_{\mathrm{B}} \exp \left[u_{j j^{\prime}}(0)\right] \exp \left[u_{j j^{\prime}}\left(t-t^{\prime}\right)\right]\right\}$ are the natural difference frequency experienced within the uncoupled $S$ and the correlation function reflecting its weak coupling to the B. If the latter is the heat bath with noninteracting harmonic oscillators (phonon normal modes), then

$$
u_{j j^{\prime}}(\tau)=\sum_{\lambda} g_{j j^{\prime}}^{(\lambda)}\left[\beta_{\lambda}^{+} \exp \left(-i \omega_{\lambda} \tau\right)-\beta_{\lambda} \exp \left(i \omega_{\lambda} \tau\right)\right]
$$

So

$$
Q_{j j^{\prime}}(\tau)=\exp \left(-D_{j j^{\prime}}\right) Y_{j j^{\prime}}(\tau)
$$

where $D_{j j^{\prime}}=\sum_{\lambda}\left(g_{j j^{\prime}}^{(\lambda)}\right)^{2}\left[2 n\left(\omega_{\lambda}\right)+1\right]$ is the DebyeWaller factor and

$$
\begin{aligned}
& Y_{j j^{\prime}}(t)=\int_{-\infty}^{\infty} d \omega \prod_{\lambda} \exp (i \omega t) \\
& \times \sum_{q(\lambda)=-\infty}^{\infty} I_{|q(\lambda)|}\left(z_{\lambda}\right)\left[\frac{n\left(\omega_{\lambda}\right)}{n\left(\omega_{\lambda}\right)+1}\right]^{q(\lambda) / 2} \delta\left(\omega-\sum_{\lambda} q(\lambda) \omega_{\lambda}\right)
\end{aligned}
$$

defines the time-dependence of the correlation function $Q_{j j^{\prime}}\left(t-t^{\prime}\right)$ in (12) with $I_{q}(z)$ being the modified Bessel function and $n(\omega)=$ 


\section{I. TESLENKO, O. L. KAPITANCHUK}

$\left[\exp \left(\hbar \omega / k_{\mathrm{B}} T\right)-1\right]^{-1}$. The coupling to the environment phonons is concentrated in the parameter $z_{\lambda} \equiv 2\left(g_{j j^{\prime}}^{(\lambda)}\right)^{2} \sqrt{n\left(\omega_{\lambda}\right)\left[n\left(\omega_{\lambda}\right)+1\right]}$, while the $q(\lambda)$ index indicates the number of phonons of the $\lambda$ th mode that accompanies the transition, with $\sum_{\lambda} q(\lambda) \geq 1$ since the minimal number of phonons is equal to 1 . But if the nuclear displacements along $\lambda$ th normal coordinate in (15) are small, then $z_{\lambda} \ll 1$. This allows one to use the asymptote $I_{|q|}(z) \approx(z / 2)^{|q|} /|q|$ ! pointing to a singlephonon process as the main contribution to transition. Hence, setting $I_{0}(z \ll 1) \approx 1$ and $I_{1}(z \ll 1) \approx(z / 2)$, and regarding the other terms in (16) as insignificant we reduce it to

$$
Y_{j j^{\prime}}(t)=\sum_{\lambda}\left(g_{j j^{\prime}}^{(\lambda)}\right)^{2} R_{\lambda}(t)
$$

where

$$
R_{\lambda}(t)=n\left(\omega_{\lambda}\right) \exp \left(i \omega_{\lambda} t\right)+\left[n\left(\omega_{\lambda}\right)+1\right] \exp \left(-i \omega_{\lambda} t\right)
$$

is the respective one-phonon correlation function. Since for this case $\exp \left(-D_{j j^{\prime}}\right) \approx 1$, one specifies the stochastic kinetic equations (11), (12) by a stationary single-phonon kernel

$$
\begin{aligned}
Q_{j j^{\prime}}\left(t-t^{\prime}\right) & =\sum_{\lambda}\left(g_{j j^{\prime}}^{(\lambda)}\right)^{2}\left\{n\left(\omega_{\lambda}\right) \exp \left[i \omega_{\lambda}\left(t-t^{\prime}\right)\right]\right. \\
& \left.+\left[n\left(\omega_{\lambda}\right)+1\right] \exp \left[-i \omega_{\lambda}\left(t-t^{\prime}\right)\right]\right\} .
\end{aligned}
$$

This corresponds to the using in (3) of a bilinear (onephonon) coupling of the $\mathrm{S}$ to the $\mathrm{E}$ of the form

$$
V=\sum_{j j^{\prime}}\left(\delta_{j j^{\prime}}-1\right) \sum_{\lambda} \chi_{j j^{\prime}}^{\lambda}\left(\beta_{\lambda}^{+}-\beta_{\lambda}\right)|j\rangle\left\langle j^{\prime}\right|
$$

instead of the nonlinear in general (multi-phonon) form of (5), with $\chi_{j j^{\prime}}^{\lambda}=V_{j j^{\prime}} g_{j j^{\prime}}^{(\lambda)}$ being the nonadiabatic (transient) coupling parameters provided that the energy balance within the whole system holds at every time instant. Moreover, in the steady-state limit for transitions between the random fluctuating energy levels at transient times $\Delta t \geq \tau_{\mathrm{tr}}$ far larger than stochastic times so as $\tau_{\mathrm{tr}} \gg \tau_{\mathrm{st}}$, the two-time randomization of a stochastic functional (13) in the stochastic equation (11), as well as in the stochastic kernel (12), is reduced to the one-time stochastic behavior. This allows one to assume in (12) a stationary approximation for the kernels

$$
\begin{aligned}
G_{j j^{\prime}}\left(t, t^{\prime}\right) & =G_{j j^{\prime}}(t, t-\tau) \approx G_{j j^{\prime}}(\tau) \\
& =\sum_{\lambda}\left|\chi_{j j^{\prime}}^{\lambda}\right|^{2} f_{j j^{\prime}}(\tau) R_{\lambda}(\tau) \exp \left(i \Delta E_{j j^{\prime}} \tau / \hbar\right)
\end{aligned}
$$

and instead (11) use the coarse-grained stochastic kinetic equation for the ensemble-averaged populations of states of the $\mathrm{S}$

$\dot{p}_{j}(t)=-\sum_{j^{\prime}} \int_{0}^{t} d \tau\left[G_{j j^{\prime}}(\tau) p_{j}(t-\tau)-G_{j^{\prime} j}(\tau) p_{j^{\prime}}(t-\tau)\right]$.

\section{MASTER EQUATION FOR STOCHASTICALLY AVERAGED POPULATIONS}

The integral differential kinetic equation for a coarsegrained evolution of populations (22) is averaged with respect to an ensemble of stochastic trajectories, so assigning the slowest times $\tau_{\mathrm{tr}} \gg \tau_{\mathrm{st}}$ to the occurrence of nonadiabatic (transient) processes for relaxation transitions between the adiabatic (steady-state) levels of the S. As such, at the faster stationary times $\Delta t \approx \tau_{\mathrm{st}}$, any relaxation transitions between the levels of the $\mathrm{S}$ accompanied by the creation or annihilation of phonons in the B might occur. However, effectively, adiabatically fluctuating levels would exhibit the stochastic dynamics. It is thus necessary to carry out an average over the realizations of stochastic trajectories for populations (22) (usually designated as $\langle\langle\ldots\rangle\rangle$ ), to find the averaged populations of the levels

$$
P_{j}(t)=\left\langle\left\langle p_{j}(t)\right\rangle\right\rangle
$$

as well as the averaged probabilities of transitions between the levels $W_{j j^{\prime}}$.

In order to do this task correctly, one needs to treat a non-Markovianity of integrands in (22) with providing an explicit averaging of the involved stochastic functionals $\left\langle\left\langle G_{j j^{\prime}}(\tau) p_{j}(t-\tau)\right\rangle\right\rangle$. The situation becomes simpler when adiabatic energy level shifts in the $\mathrm{S}$ are stationary. In this case, making an average on the transition time scale $\tau_{\text {st }} \ll \Delta t \leq \tau_{\text {tr }}$ factorizes the respective quantities on the right-hand side of (22), for instance, as

$$
\left\langle\left\langle f_{j j^{\prime}}(\tau) p_{j}(t-\tau)\right\rangle\right\rangle=F_{j j^{\prime}}(\tau) P_{j}(t) .
$$

This means that in the second-order perturbation theory, a non-Markovianity of the population of the metastable level does not imply itself giving $P_{j}(t-\tau) \approx P_{j}(t)$. Moreover, an arisen stochastically averaged functional

$$
F_{j j^{\prime}}(\tau)=\left\langle\left\langle f_{j j^{\prime}}(\tau)\right\rangle\right\rangle=\left\langle\left\langle\exp \left[i \int_{0}^{\tau} \Omega_{j j^{\prime}}(t) d t\right]\right\rangle\right\rangle
$$

becomes exactly calculable, e.g., for the discrete stochastic processes [6,7] and a Gaussian white noise [11]. In such cases, (25) reduces to the simple form

$$
F_{j j^{\prime}}(\tau)=\exp \left(-\gamma_{j j^{\prime}} \tau\right)
$$

where $\gamma_{j j^{\prime}}=\gamma_{j^{\prime} j}$ is the symmetric effective level halfwidth associated with a friction coefficient for the movement of particles within the $\mathrm{S}$ when modeled to be open to the B.

Using (23)-(26) reduces the integral differential equation (22) to the master evolution equation

$$
\dot{P}_{j}(t)=-P_{j}(t) \sum_{j^{\prime} \neq j} W_{j j^{\prime}}+\sum_{j^{\prime} \neq j} P_{j^{\prime}}(t) W_{j^{\prime} j}
$$

where populations (23) of the energy levels of the $\mathrm{S}$ are nonconserved $0 \leq \sum_{j} P_{j}(t) \leq 1$ on the longest, nonadiabatic (transient) time scale $\Delta t \geq \tau_{\text {tr }}$ while partially normalized on the much shorter, adiabatic (stochastic) time scale $\Delta t \approx \tau_{\text {st }} \ll \tau_{\text {tr }}$, whereas the probabilities of transitions from level $j$ to level $j^{\prime} \neq j$ (stochastically averaged transition rate constants) 


$$
W_{j j^{\prime}}=\frac{2}{\hbar^{2}} \int_{0}^{\infty} d \tau \exp \left[\left(\frac{i}{\hbar} \Delta E_{j j^{\prime}}-\gamma_{j j^{\prime}}\right) \tau\right] \sum_{\lambda}\left|\chi_{j j^{\prime}}^{\lambda}\right|^{2}\left\{n\left(\omega_{\lambda}\right) \exp \left(i \omega_{\lambda} \tau\right)+\left[1+n\left(\omega_{\lambda}\right)\right] \exp \left(-i \omega_{\lambda} \tau\right)\right\}
$$

appear to be time-independent at all times. Formally, $W_{j j^{\prime}}(28)$ can be calculated from the Fermi golden rule [12] while paying attention not to summing over a dense set of final levels but rather to weighting over the stochastic trajectories for transitions with different stationary contributions concentrated in the form of their amplitude and intensity distributions. However, determining the order of carrying out the calculation of (28) entails some difficulties. At first, one needs to obtain a sum over the number $\Lambda$ of phonon modes $\lambda=1, \ldots, \Lambda$, which in general is infinite and may thus diverge, and only then evaluate the integral over time $\tau$ in the interval from 0 to $\infty$, given $\Delta E_{j j^{\prime}}$ and $\gamma_{j j^{\prime}}$. Since in (23)-(26) one provides an account of random additions to energy levels with the single parameters $\gamma_{j j^{\prime}}$ without using the perturbation theory [6-9,11], this allows for the analysis of different regimes for transitions dependently on the values of stochastic intensity $\gamma_{j j^{\prime}}$ and dynamic amplitude $\left|\Delta E_{j j^{\prime}}\right|$ parameters for the $\mathrm{S}$ in some limiting cases. Therefore, making necessary assumptions about the relation of these parameters and assuming the dependence of nonadiabatic couplings $\left|\chi_{j j^{\prime}}^{\lambda}\right|^{2}$ on $\lambda$ for different $j \neq j^{\prime}$ will be sufficient for changing the orders of summation and integration in (28) to represent the calculations most accurately.

On the other hand, transition rate constants (28) can be used to set up a transition rate matrix $\tilde{W}$ with the components [13]

$$
\tilde{W}_{j j^{\prime}}=W_{j j^{\prime}}-\delta_{j j^{\prime}} \sum_{l=0}^{J} W_{l j}
$$

This allows one to write a master equation (27) for the state vectors $\mathbf{P}(t)=\operatorname{col}\left(P_{0}(t), \ldots, P_{J}(t)\right)$ in the generalized matrix form

$$
\frac{d \mathbf{P}(t)}{d t}=\tilde{W} \mathbf{P}(t)
$$

By its physical sense, Eq. (30) is nothing else than a result of procedure of multiple projection of the Liouvillevon Neumann quantum evolution equation (6) onto the specified multidimensional states space in the imaginary time space. The projection is made sequentially using the following ordering: first, onto the space of the diagonal elements of the nonequilibrium density matrix (10) (corresponds to a vector representation of density matrix by retaining only its diagonal elements and avoiding off-diagonal ones in terms of diagonalization); then, onto the microscopic energy-level population space (11), (22) (corresponds to the integrating out of normal vibrations of the heat bath); further, onto the stochastically averaged state population space (27) (corresponds to an averaging over the random fluctuations of microscopic energy levels); and finally, onto the aggregated population space (corresponds to involving the Markov chain modular states given in (46), Section 5). In this context, transition rate matrix $\tilde{W}$ in (30) will coincide with the Liouville superoperator $L(t)$ in (6) provided that the imaginary time transformation $t \rightarrow i t$ is applied. In general, if the $\tilde{W}(29)$ or $-i L(t)$ (6) cannot be decomposed into the block form, then the finite space system (27) has a uniquely defined equilibrium state $\mathbf{P}^{\text {eq }}=\mathbf{P}(t \rightarrow \infty)$ for which

$$
\left.\frac{d \mathbf{P}(t)}{d t}\right|_{\mathbf{P}(t)=\mathbf{P}^{\mathrm{eq}}}=\left.\frac{d \mathbf{P}(t)}{d t}\right|_{t \rightarrow \infty}=0
$$

This implies that $\tilde{W}$ has a single zero eigenvalue $\lambda_{0}=0$, while all the other eigenvalues are real and negative [14]. If to use the independent kinetic modes (eigenmodes) being in fact the Debye relaxation rates $\left\{r_{j=0, \ldots, J}\right\}$ and to arrange them in ascending order instead of in order of increasing the absolute algebraic value of their eigenvalues $\left\{\left|\lambda_{j=0, \ldots, J}\right|\right\}$, then all these eigenmodes become real and non-negative, that is $0=r_{0} \leq r_{1} \leq \ldots \leq r_{J}$. Therefore, within the $\mathrm{S}$, the set of relaxation rates $\left\{r_{j}\right\}$ will be complete and, by definition, correspond to the characteristic inverse transient times $\left\{\tau_{\mathrm{tr}}^{(j)}\right\}^{-1}$ determined by transition probabilities (28) on the longest time scale.

However, in the $\mathrm{S}$, there must always exist the fundamentally shortest time scale too, termed chaotic, namely $\Delta t \approx \tau_{\text {ch }} \ll \tau_{\text {st }} \ll \tau_{\text {tr }}$, on which the ergodically mixed sets (or modules) of quasi-isoenergetic (i.e., nearly degenerate) energy levels are formed [7,8]. Just on this time scale, the microscopic structure of the Hamiltonians of the whole closed system (1)-(5) conditioned with the factorization of its density matrix (7) is created. Furthermore, an attribute "chaotic" should mean that, to proceed further from the very short times $t \ll \tau_{\text {ch }}$ at which the energy levels of the $\mathrm{S}$ were intrinsically highly correlated to chaotic times $t \approx \tau_{\mathrm{ch}}$, one would reduce all the groups of strongly interacted quasi-degenerate states to the effectively uncoupled ones and then chaotize them in accordance with some pre-existed distribution (for example, a Gaussian). Therefore, that time could be expected to be close to an interaction/encounter/scattering time or to the time of establishment of local equilibrium within the $\mathrm{S}$ with a highest physical speed and, thus, has to directly relate to the notion of quasi-averages in statistical mechanics [15-18]. Meanwhile, for the coherent dynamics of the $\mathrm{S}$, initial chaotization implies that individual positions of the energy levels become not timeindependent but rather statistically randomized according to the some stochastic distribution completed at time $t \approx \tau_{\text {st }}$. There is, no doubt, a situation of modeling such positions of levels microscopically unchanged during the elapsed times between two successive encounters. Consequently, if microscopically approaching the problem as in (1)-(4), one will have to additionally stochastize the eigenenergy levels of the Hamiltonian of the $\mathrm{S}$ on the 


\section{I. TESLENKO, O. L. KAPITANCHUK}

stochastic coupling time scale (i.e., the inverse encounter rate scale) which must thus be largely extended from both the corresponding relaxation transition time and the pre-supposed random chaotic time such as $\tau_{\text {st }} \ll \tau_{\text {tr }}$ and $\tau_{\text {st }} \gg \tau_{\text {ch }}$, respectively, or, jointly, as $\tau_{\text {ch }} \ll \tau_{\text {st }} \ll \tau_{\text {tr }}$.

The aforementioned arguments allow one to provide a decomposition between the $J+1$ energy levels, initially introduced for the $\mathrm{S}$, with a combination of them in the $M$ modules $m=1, \ldots, M$, to be the transient ones, and singling out the one module $m=$ 0 , to be an absorbing one. As such, each $m$ th module consists of $\mu_{m}$ energy levels. But all of the levels are in turn decomposed into the corresponding modules $\left\{\left(j_{0}^{\left(1_{0}\right)}, j_{0}^{\left(2_{0}\right)}, \ldots, j_{0}^{\left(\mu_{0}\right)}\right),\left(j_{1}^{\left(1_{1}\right)}, j_{1}^{\left(2_{1}\right)}, \ldots, j_{1}^{\left(\mu_{1}\right)}\right), \ldots\right.$, $\left.\left(j_{M}^{\left(1_{M}\right)}, j_{M}^{\left(2_{M}\right)}, \ldots, j_{M}^{\left(\mu_{M}\right)}\right)\right\}$ bearing the respective indexes $\left(j_{m}^{\left(1_{m}\right)}, j_{m}^{\left(2_{m}\right)}, \ldots, j_{m}^{\left(\mu_{m}\right)}\right)$ for every $m$, such that to conserve the total number of levels

$$
\sum_{m=0}^{M} \mu_{m} \equiv \sum_{m=0}^{M} \sum_{j_{m}=1_{m}}^{\mu_{m}} 1=\sum_{j=0}^{J} 1=J+1
$$

Moreover, without the loss of generality, the level degeneracy of an absorbing module can always be made high enough to correctly justify the very absorbing property of exactly the module $m=0$. But, of course, this is the case only if to reasonably substantiate an assumption noted above on the bounding from below of a physical speed limit for the chaotization rate $\tau_{\mathrm{ch}}^{-1}$ of being much higher than that corresponding to the stochastization $\tau_{\mathrm{ch}}^{-1} \gg \tau_{\mathrm{st}}^{-1}$ and transition $\tau_{\mathrm{ch}}^{-1} \gg \tau_{\mathrm{st}}^{-1} \gg \tau_{\mathrm{tr}}^{-1}$ rates, respectively, characteristic for the $\mathrm{S}$. This straightforwardly points to a concern to calculate the rate constant (28) of relaxation transitions between different levels most rigorously.

\section{TRANSITION RATE CONSTANTS}

Expression (28) for the probability of environmentinduced transitions between the fluctuating energy levels of the $\mathrm{S}$ is characteristic for the approaches of describing a nonadiabatic relaxation in the different nonequilibrium condensed phase systems $[6,9,12]$. Here, it appears as a result of averaging over the random fluctuations completed at stochastic times $\tau_{\text {st }}$ and is approved for the calculation of rate constants at far longer relaxation transitions times $\tau_{\text {tr }}$. Importantly, the same expression (28) holds at much shorter chaotic times too, obeying a natural hierarchy with respect to the other two $\tau_{\text {ch }} \ll \tau_{\text {st }} \ll \tau_{\text {tr }}$. Any information about the time scales involved is neither lost nor filtered out but rather is correctly accounted for in the master equation (27) for populations (23) by the corresponding averages - equilibrium occupation phonon numbers $n\left(\omega_{\lambda}\right)$ formed in the $\mathrm{B}$ at chaotic times $\tau_{\mathrm{ch}}$, intensities $\gamma_{j j^{\prime}}$ of the stationary adiabatic fluctuations of energy levels established in the $\mathrm{S}$ at stochastic times $\tau_{\mathrm{st}}$, and parameters $\left|\chi_{j j^{\prime}}^{\lambda}\right|^{2}$ of the environment-induced (assisted by the phonons of the B) nonadiabatic interaction between the $\mathrm{S}$ and the $\mathrm{B}$ responsible for the relaxation processes occurred at the transition times $\tau_{\text {tr }}$, respectively. Moreover, within the steady-state approximation (23)-(26), one can reduce this expression to the timeconvolutionless form. Indeed, taking on the integral over $\tau$ in (28) with making an account of (23)-(26) yields

$$
\begin{aligned}
W_{j j^{\prime}} & =\frac{2 \pi}{\hbar^{2}} \sum_{\lambda}\left|\chi_{j j^{\prime}}^{\lambda}\right|^{2}\left\{n\left(\omega_{\lambda}\right) \Lambda_{j j^{\prime}}^{(+)}\left(\omega_{\lambda}\right)\right. \\
& \left.+\left[1+n\left(\omega_{\lambda}\right)\right] \Lambda_{j j^{\prime}}^{(-)}\left(\omega_{\lambda}\right)\right\}
\end{aligned}
$$

where $\Lambda_{j j^{\prime}}^{( \pm)}\left(\omega_{\lambda}\right)=\gamma_{j j^{\prime}}\left\{\pi\left[\gamma_{j j^{\prime}}^{2}+\left(\omega_{\lambda} \pm \Delta E_{j j^{\prime}} / \hbar\right)^{2}\right]\right\}^{-1}$ is the high-frequency bound of stochastic field generated Lorentzian [7]. However, to further take on the sum over $\lambda$ in (33) requires knowing the energy level spectrum of the $\mathrm{S}$ as well as the most relevant environment-induced relaxation parameters. Therefore, if focusing on a simple but correct description of relaxation processes at chaotic times including the stochastic and transient ones, one should be interested mainly in the calculable cases for transition probabilities (33). Also, in order to be associated with the observable reaction rates these probabilities would be well interpretable.

To be concrete in concerning with a calculation of (33) on the different time scales, let us assume that the $\mathrm{S}$ represents the some flexible, intrinsically disordered nanosystem able to make as fast diffusive jumps over its adiabatic multidimensional energy surfaces by turning, coiling, bending, twisting and looping as well slower relaxation transitions between these surfaces of forming the covalent and non-covalent bonds [19-22]. This leads to reducing the $\mathrm{S}$ to a system having that structure of the energy level spectrum which is composed of the number of narrow quasi-isoergic bands. These bands are only occasionally degenerated, not because of overlapping the respective nearest-neighboring states between each other, but owing to the great number of near-degenerate weakly interacting configurations of states. The latter are normally present around the functional groups of the $\mathrm{S}$ in the interior of inherently unstructured, disordered regions $[19,20]$, as well as in the bulk of the $\mathrm{B}$ when considering it to be in the open contact to the $\mathrm{S}[8,9,22]$.

Let us confine ourselves to considering two important realizations of the $\mathrm{S}$ in only the limiting cases. Namely: the case of nonadiabatic (nonstationary) transitions with

$$
\left|\Delta E_{j j^{\prime}}\right| / \hbar \approx \omega_{\lambda} \gg \gamma_{j j^{\prime}} \rightarrow+0
$$

and the case of adiabatic (stationary) transitions with

$$
\gamma_{j j^{\prime}} \gg \omega_{\lambda} \geq\left|\Delta E_{j j^{\prime}}\right| / \hbar \rightarrow+0
$$

Since the relation for thermal intensity of stochastic fluctuations at room temperature $[6,7]$ is

$$
\gamma_{j j^{\prime}}=k_{\mathrm{B}} T / \hbar
$$

the cases (34) and (35) directly correspond to the quantum

$$
\left|\Delta E_{j j^{\prime}}\right| \gg k_{\mathrm{B}} T
$$


and classical

$$
\left|\Delta E_{j j^{\prime}}\right| \ll k_{\mathrm{B}} T
$$

limits, respectively. Furthermore, irrespective of the temperature, one can introduce a rate speed limit for symmetric frequency-independent asymptotes $\Im_{j j^{\prime}}=\Im_{j^{\prime} j} \equiv$ $\Im_{j j^{\prime}}\left(\omega_{\lambda} \approx\left|\Delta E_{j j^{\prime}}\right| / \hbar\right)$ of the one-phonon spectral function

$$
\begin{array}{r}
\Im_{j j^{\prime}}=\lim _{\omega_{\lambda} \rightarrow\left|\Delta E_{j j^{\prime}}\right| / \hbar}\left(2 \pi / \hbar^{2}\right) \sum_{\lambda}\left(\left|\chi_{j j^{\prime}}^{\lambda}\right|^{2} / \omega_{\lambda}\right)= \\
=\left(2 \pi / \hbar^{2}\right) \sum_{\lambda}\left|\chi_{j j^{\prime}}^{\lambda}\right|^{2} \delta\left(\omega_{\lambda}-\left|\Delta E_{j j^{\prime}}\right| / \hbar\right)
\end{array}
$$

being of prime interest in any microscopic calculations on multi-level systems [13]. Hence, by introducing the generalized distribution function

$$
n\left(\Delta E_{j j^{\prime}}\right)=\left[\exp \left(\Delta E_{j j^{\prime}} / k_{\mathrm{B}} T\right)-1\right]^{-1}
$$

with the anticommutation property $n\left(\Delta E_{j j^{\prime}}\right)+$ $n\left(\Delta E_{j^{\prime} j}\right)=-1$, and the signum function

$$
\operatorname{sgn}\left(\Delta E_{j j^{\prime}}\right)=\left\{\begin{array}{r}
1, \Delta E_{j j^{\prime}}>0 \\
0, \Delta E_{j j^{\prime}}=0 \\
-1, \Delta E_{j j^{\prime}}<0
\end{array}\right.
$$

defined for all $\Delta E_{j j^{\prime}}$ including $\Delta E_{j j^{\prime}}=0$ with a convention $n(0) \operatorname{sgn}(0)=1$, one can reduce rate constant (33) to the form most appropriate for both cases above $(34),(37)$ and (35), (38):

$$
W_{j j^{\prime}}=\Im_{j j^{\prime}} n\left(\Delta E_{j j^{\prime}}\right) \operatorname{sgn}\left(\Delta E_{j j^{\prime}}\right) .
$$

This marginally correct expression is physically strict and satisfies the principle of microscopic reversibility (a condition of detailed balance)

$$
W_{j j^{\prime}}=W_{j^{\prime} j} \exp \left(\Delta E_{j j^{\prime}} / k_{\mathrm{B}} T\right)
$$

regardless of the presence in the $\mathrm{S}$ of irreversible kinetic stages, by considering the reverse rates for them to be infinitely rare or almost insignificant. Moreover, expression (42) reproduces well the very fast (often fastest) rate speed limit characteristic for activationless intraband transitions

$$
W_{j^{\prime} j}=\Im_{j j^{\prime}}
$$

with the near zero or negligibly small activation energy $E^{\text {act }} \equiv\left|\Delta E_{j j^{\prime}}\right| \ll k_{\mathrm{B}} T$, as well as the far slower Arrhenius's rate limit characteristic for activation-like interband transitions

$$
W_{j j^{\prime}}=\Im_{j j^{\prime}} \exp \left(-E^{\mathrm{act}} / k_{\mathrm{B}} T\right) \ll W_{j^{\prime} j}
$$

commonly having the much larger activation energy $E^{\text {act }} \equiv\left|\Delta E_{j j^{\prime}}\right| \gg k_{\mathrm{B}} T$.

Consequently, the classification of environmentinduced transitions between the energy levels of the $\mathrm{S}$ is as follows. At first, on the chaotic time scale $\tau_{\mathrm{ch}}$, one can provide the rates of intra-band transitions between fluctuating energy levels belonging to the every $m$ th band of the $\mathrm{S}$ with an ergodic mixing property for all the $\mu_{m}$ degenerate levels within that band, and regard each such band as the $m$ th module of levels to be combined in a single modular state characterized by one the same level of energy $E_{m}$ and dimensionality $\mu_{m}$. At second, on the stochastic time scale $\tau_{\text {st }}$, one can let these modules to be in a contact of one another in a bottleneck fashion with allowing their energies to fluctuate with an independent of $m$ thermal intensity (36). And finally, on the transient time scale $\tau_{\text {tr }}$, one can additionally endow the modules with inter-band transition rates to be the one of only two important types: the mechanistic type (44) of temperature-independent transitions and the Arrhenius's type (45) of exponentially temperature dependent transitions. The reduced master equation for evolution of the aggregated modular populations

$$
\Gamma_{m}(t)=\sum_{j_{m}=1}^{\mu_{m}} P_{j_{m}}(t)
$$

corresponding to this classification is easily derived from the general master equation (27) in the approximation (45), given condition (32), in the following form

$$
\dot{\Gamma}_{m}(t)=-\Gamma_{m}(t) \mu_{m}^{-1} \sum_{m^{\prime} \neq m} W_{m m^{\prime}}+\sum_{m^{\prime} \neq m} \Gamma_{m^{\prime}}(t) W_{m^{\prime} m} \mu_{m^{\prime}}^{-1}
$$

with

$$
W_{m m^{\prime}}=\Im_{m m^{\prime}} n\left(\Delta E_{m m^{\prime}}\right) \operatorname{sgn}\left(\Delta E_{m m^{\prime}}\right) \ll W_{j_{m} j_{m}^{\prime}} \approx W_{j_{m^{\prime}} j_{m^{\prime}}^{\prime}}
$$

being the inter-module $\left(m \neq m^{\prime} ; m, m^{\prime}=0,1, \ldots, M\right)$ transition rates significantly smaller than those for intramodule transitions, and $\Delta E_{m m^{\prime}} \equiv E_{m}-E_{m^{\prime}}$ are the re- spective inter-module energy level differences being the same for all pairs of levels $\left(j_{m} ; j_{m^{\prime}}^{\prime}\right)$ if taken from the different modules. 


\section{I. TESLENKO, O. L. KAPITANCHUK}

\section{DEFINITION OF COMPETITIVITY}

Eq. (47) represents the coarse-grained master equation for a consistent kinetic description of the time evolution of population (46) of the multidimensional, ergodically mixed modular states of the some open system (S) performing relaxation transitions between the fluctuating energy levels due to a weak coupling to the heat bath (B) on the transient time scale. The main advantage of this representation is that it allows the possibility of interpreting an absorbing Markov chain as of the irreversible environment-induced relaxation process in the nonequilibrium system interacting with the equilibrium environment. Furthermore, it correctly defines the transition rate constants (48) by admitting a controllability of their potential change in the extended limits. For example, one can easily associate an absorbing Markov state with a singled out module $m=0$ having the very high or even infinite dimensionality so that the aggregated probability of all leavings of this module is regarded exceptionally rare or infinitely uncommon with respect to entering it:

$$
\sum_{m=1}^{M} W_{m 0} \gg \sum_{m=1}^{M} W_{0 m} \rightarrow 0
$$

Also, without an offer up to correctness of equations (47), (48), one can restrict his/her attention to only the operationally relevant case, where it is possible to reset a Markov chain every time it reaches absorbing state by singling out the other module, say, module $m=M$ as the starting one for another irreversible cycle. The latter can be initiated by presupposing a dimensionality of this module to be infinite $\mu_{M}=\infty$ before the beginning of each new cycle, whereas letting this value free but finite in the interval $1 \leq \mu_{M}<\infty$ exactly after the cycle begins.

In general, an $M+1$ state Markov chain, regardless of being absorbing or recurrent, has $2 M-1$ free parameters supposed a priori independent and time-homogeneous. There are the $M$ Debye relaxation rates $r_{m=1, \ldots, M}$, the $M$ weights $A_{m}$ of exponential contributions in decomposition time series, and minus one parameter determined through the initial condition $\Gamma_{m=1, \ldots, M-1}(t=0)=0$; $\Gamma_{M}(t=0)=1[3,24]$. Since, by definition, an absorbing Markov chain is characterized by the nonconserved populations $\Gamma_{m=1, \ldots, M}(t \rightarrow \infty)=0\left(\right.$ but $\left.\Gamma_{m=0}(t \rightarrow \infty)=1\right)$, and has the relaxation rate spectrum with the real and positive roots $r_{m=1, \ldots, M}>0$ (but $r_{0}=0$ ), the populations do not oscillate. However, function $\Gamma_{M}(t)$ will be strictly monotonic while functions $\Gamma_{m=1, \ldots, M-1}(t)$ will increase, peak and decline. Therefore, for the latter, one can always introduce the amplitudes of their peaks

$$
\bar{\Gamma}_{m=1, \ldots, M-1}=\Gamma_{m=1, \ldots, M-1}\left(t=t_{m}^{p}\right)
$$

to find them from the equations

$$
\frac{d \Gamma_{m=1, \ldots, M-1}(t)}{d t}=0
$$

for defining the corresponding peak times $t_{m}^{p}$, respectively.

To implement the properties above in a straightforward representation, one can avoid any kinetic cycles in an absorbing Markov chain by reducing it to a linear chain with no loop configurations and also use the leading order for connecting transitions between the only adjacent states $\langle m\rangle$, each of dimensionality $\mu_{m}$ and energy separation from other states $\Delta E_{m m^{\prime}} \equiv E_{m}-E_{m^{\prime}}$ :

$$
\langle M\rangle \underset{W_{M-1 M}}{\stackrel{W_{M M-1}}{\longrightarrow}}\langle M-1\rangle \cdots\langle 3\rangle \underset{W_{23}}{\stackrel{W_{32}}{\longrightarrow}}\langle 2\rangle \underset{W_{12}}{\stackrel{W_{21}}{\longrightarrow}}\langle 1\rangle \stackrel{W_{10}}{\longrightarrow}
$$

In a linear absorbing Markov chain above, the $M$ states are characterized exactly by the $2 M-1$ independent leading-order rate constants $W_{m m^{\prime}}(48)$ can potentially vary in arbitrary limits. The complete set of them forms both the necessary and sufficient conditions to define kinetics of the irreversible evolution of state populations $\Gamma_{m=1, \ldots, M}(t)$ in the operational framework, given initial conditions. Particularly for (52), one determines the response laws which establish the functional relationships $\bar{\Gamma}_{m}\left(W_{m m^{\prime}}\right)$, as well as $r_{m}\left(W_{m m^{\prime}}\right)$, between the corresponding variations in growth of transition rates $W_{m m^{\prime}}$ and the variations of gain in population peaks $\bar{\Gamma}_{m}$, as well as the variations in change of relaxation rates $r_{m}$, respectively. Since the problem (47)-(52) is transcendent, such relationships remind of nonanalytic or fractal response laws often included in the kinetic models a priori $[25,26]$. But contrary to this, in the aforedescribed microscopic approach (23)-(28) to an absorbing Markov chain problem (50)-(52), the same relationships may be involved analytically.

To correctly define the competitivity between different states of an absorbing Markov chain (52), we realize that every state, excluding the initial one, is in competition with other states. These, in accordance with the physical laws of evolution, are all, themselves, directed to compete between each other in terms of gaining their population peaks for growth of logarithm of the supply rates provided effective energy input to them. Furthermore, each state prefers to maximize respective gain in a range of the most stable increase of population peak, that is, in the vicinity of inflection point of the functional dependence $\bar{\Gamma}_{m}\left[\log \left(W_{m m^{\prime}}\right)\right]$, where the slope of the log odds of population peak with respect to $\log \left(W_{m m^{\prime}}\right)$ becomes maximal [9], and vice versa. For these reasons, we may define the competitivity of state $\langle m\rangle$ as its ability to provide a control of the energy supply input factor $\log \left(W_{m^{\prime} m^{\prime \prime}}\right)\left(m, m^{\prime}, m^{\prime \prime}=1, \ldots, M-1 ; m^{\prime} \neq m^{\prime \prime}\right)$ by the following general relation

$$
\begin{aligned}
\eta_{m^{\prime} m^{\prime \prime}}^{(m)} & =\frac{d \log \left(W_{m^{\prime} m^{\prime \prime}}\right)}{d \log \left[\bar{\Gamma}_{m} /\left(\bar{\Gamma}_{m}^{(\infty)}-\bar{\Gamma}_{m}\right)\right]} \\
& =\frac{d \log \left(W_{m^{\prime} m^{\prime \prime}}\right)}{d \log \left[\overline{\bar{\Gamma}}_{m} /\left(1-\overline{\bar{\Gamma}}_{m}\right)\right]}
\end{aligned}
$$

where $\bar{\Gamma}_{m}^{(\infty)}=\bar{\Gamma}_{m}\left(W_{m^{\prime} m^{\prime \prime}} \rightarrow \infty\right)$ is the saturation level of the sigmoid dependence $\bar{\Gamma}_{m}\left[\log \left(W_{m^{\prime} m^{\prime \prime}}\right)\right]$ and 
$\overline{\bar{\Gamma}}_{m} \equiv \bar{\Gamma}_{m} / \bar{\Gamma}_{m}^{(\infty)}$. As we see, the procedure of determining the competitivity $\eta_{m^{\prime} m^{\prime \prime}}^{(m)}$ is as follows. First of all, it is necessary to solve the master equation (47) for a chain (52) with the arbitrary transition rates $W_{m m^{\prime}}$, given initial conditions $\Gamma_{m=1, \ldots, M-1}(t=0)=0 ; \Gamma_{M}(t=0)=1$. Further, it requires find the peaks for the functions $\Gamma_{m=1, \ldots, M-1}(t)$ attained at peak times $t_{m}^{p}$ (50), (51), build for these peaks the sigmoid curves $\bar{\Gamma}_{m}\left[\log \left(W_{m^{\prime} m^{\prime \prime}}\right)\right]$ of interest, normalize those curves to unity and calculate the log odds of thus normalized peaks $\overline{\bar{\Gamma}}_{m}$ (53). Then, it needs vary both the transition rates $W_{m^{\prime} m^{\prime \prime}}$ and the states $\langle m\rangle$ in $(52)$ to associate the competitivity $\eta_{m^{\prime} m^{\prime \prime}}^{(m)}$ found in (53) with the involved triples $\left(m, m^{\prime}, m^{\prime \prime} \neq m^{\prime}\right)$. And finally, it allows to plot the resulting multidimensional competitivity landscape for variation of $\eta_{m^{\prime} m^{\prime \prime}}^{(m)}$ in the space of $2 M-1$ rate variables $W_{m^{\prime} m^{\prime \prime}}$ to be set as the free independent parameters in describing the competitivity of every state each time to quantify it.

Obviously, in the general case of very large $M$, the aforementioned procedure comprises the complex hardto-solve problem, also known as the number-of-states problem [27]. Apart from some exceptions [28], the Lyapunov-stable solution to this problem always exists and is unique. Nonetheless, to perform an exact calculus of kinetics of the $\mathrm{S}$ is often unnecessary and expensive computationally. Instead, one intends to understand how potentially many states are correlated such that to shorten a description of microscopically reversible transitions between the recurrent Markov states and macroscopically irreversible decay to an absorbing Markov state to only a few effective transitions. And how is to control the competitivity of states, so that to be consistent in relating between the normalized peaks of population and the thermodynamic quasi-averages like states' level energy and dimensionality. Note that this corresponds to shortening the description of nonequilibrium systems originally formulated by Bogoliubov as a principle of weakening of correlations in solving the initial value problem, and is equivalent to a procedure of contracting of the number of independent variables in the boundary condition problem, both basing on the idea of a hierarchy of characteristic relaxation rates in the system [15-18].

To conclude, a continuous time multidimensional absorbing Markov chain can consistently be represented by the process of evolution of a nonequilibrium system with adiabatically fluctuating energy levels, occurring due to a weak nonadiabatic coupling to the equilibrium environment on the different time scales. For this system, it is possible to correctly define the competitivity of states as an inverse slope of log odds of normalized peaks of state population with respect to log of growth in various input transition rates directed to population gain of states (53). However, the procedure of quantitatively determining the competitivity in the general case is very complicated and needs further work. More specifically, in the next paper we will show that, at least for a twostate absorbing Markov chain, the competitivity cannot be less than unity or "negative" but rather be larger than unity or "positive" yet always bounded from above by a particular value.

\section{ACKNOWLEDGEMENTS}

The present work was partially supported by The National Academy of Sciences of Ukraine (project No. 0110U007542).
[1] W. E, T. Li, E. Vanden-Eijnden, Proc. Natl. Acad. Sci. USA 105, 7907 (2008).

[2] J. D. Chodera et. al, J. Chem. Phys. 126, 155101 (2007).

[3] W. J. Bruno, J. Yang, J. E. Pearson, Proc. Natl. Acad. Sci. USA 102, 6326 (2005).

[4] G. Bolch, S. Greiner, H. de Meer, K. S. Trivedi, Queueing Networks and Markov Chains: Modeling and Performance Evaluation with Computer Science Applications (Wiley, New York, 2006).

[5] C. Baier, J.-P. Katoen, Principles of Model Checking (MIT Press, Cambridge, 2008).

[6] V. I. Teslenko, E. G. Petrov, A. Verkhratsky, O. A. Krishtal, Phys. Rev. Lett. 104, 178105 (2010).

[7] E. G. Petrov, V. I. Teslenko, Chem. Phys. 375, 243 (2010).

[8] V. I. Teslenko, O. L. Kapitanchuk, Ukr. J. Phys. 57, 573 (2012).

[9] V. I. Teslenko, O. L. Kapitanchuk, Int. J. Mod. Phys. B 27, 1350169 (2013).

[10] T. Holstein, Ann. Phys. 8, 343 (1959).

[11] R. F. Fox, Phys. Rep. 48, 179 (1978).
[12] A. Nitzan, Chemical Dynamics in Condensed Phases (Oxford University Press, Oxford, New York, 2006).

[13] M. A. Miller, J. P. K. Doe, D. J. Wales, Phys. Rev. E 60, 3701 (1999).

[14] N. G. van Kampen, Stochastic Processes in Physics and Chemistry (North-Holland, Amsterdam, 1981).

[15] N. N. Bogoliubov, Lectures on Quantum Statistics. Vol. 1 (Gordon and Breach, New York, 1967).

[16] N. N. Bogoliubov, Lectures on Quantum Statistics. Vol. 2 (Gordon and Breach, New York, 1970).

[17] N. N. Bogoliubov, N. N. Bogoliubov, Jr., Introduction to Quantum Statistical Mechanics, 2nd ed. (World Scientific, Singapore, 2009).

[18] A. L. Kuzemsky, Int. J. Mod. Phys. B 24, 835 (2010).

[19] M. D. Fayer, Annu. Rev. Phys. Chem. 52, 315 (2001).

[20] D. D. Boehr, H. J. Dyson, P. E. Wright, Chem. Rev. 106, 3055 (2006).

[21] M. K. Prakash, R. A. Marcus, Proc. Natl. Acad. Sci. USA 104, 15982 (2007).

[22] G. Fleming, M. Ratner, Physics Today 61, 28 (2008).

[23] A. J. Leggett et al., Rev. Mod. Phys. 59, 1 (1987). 
[24] R. Metzler, J. Klafter, J. Jortner, Proc. Natl. Acad. Sci. USA 96, 11085 (1999).

[25] M. O. Vlad, F. Moran, V. T. Popa, S. E. Szedlacsek, J. Ross, Proc. Natl. Acad. Sci. USA 104, 4798 (2007).
[26] A. Rovenchak, J. Phys. Stud. 17, 2001 (2013).

[27] P. Suppes, J. Math. Psychol. 6, 327 (1969).

[28] W. M. L. Holcombe, Algebraic Automata Theory (Cambridge University Press; Cambridge, 1982).

\title{
ВИЗНАЧЕННЯ КОНКУРЕНТОСПРОМОЖНОСТІ АДСОРБУЮЧОГО ЛАНЦЮГА МАРКОВА
}

\author{
В. І. Тесленко, О. Л. Капітанчук \\ Інститут теоретичної фізики ім. М. М. Боголюбова НАН Украӥни, \\ вул. Метрологічна, 14б, Київ, 03680, Украӥна
}

\begin{abstract}
Сформульовано проблему визначення конкурентоспроможності для різних станів адсорбуючого ланцюга Маркова за неперервного розвинення в часі. Стани ланцюга співвідносяться з агрегатами для локальнорівноважних флуктуючих енергетичних рівнів, що виникають унаслідок адіабатичних взаємодій усередині нерівноважної системи, яка, своєю чергою, еволюціонує за наявності неадіабатичного зв'язку з рівноважним оточенням. Використовуючи послідовний мікроскопічний підхід до опису еволюцї матриці густини повної системи, ми спершу редукуємо відповідне загальне рівняння Ліувілля-фон Ноймана до основного рівняння для діагональних елементів матриці густини нерівноважної системи, відтак даємо розрахунок хаотичних та стохастичних середніх за певними розподілами енергетичних рівнів і нарешті виводимо кінетичне рівняння для заселеності агреґатних станів адсорбуючого ланцюга Маркова. Такий розгляд надає визначенню конкурентоспроможності різних станів вигляду зворотного нахилу функції логарифма переваг для нормованих піків їх агрегатних заселеностей щодо логарифма відповідних вхідних констант швидкостей переходів, що змінюються в широких границях.
\end{abstract}

